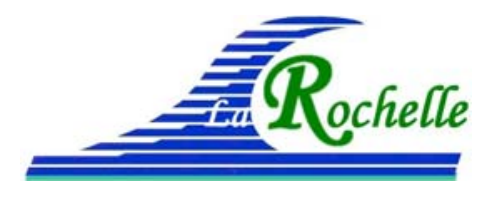

XVèmes Journées Nationales Génie Côtier-Génie Civil

La Rochelle, 29 au 31 mai 2018

DOI:10.5150/jngcgc.2018.074

(C) Editions Paralia CFL

disponible en ligne - http://www.paralia.fr - available online

\title{
Élaboration de la cartographie nationale des ouvrages et aménagements littoraux
}

\section{François HÉDOU ${ }^{1}$, Amélie ROCHE ${ }^{1}$, Marie CAOUISSIN ${ }^{1}$, Sabine MORAUD ${ }^{2}$, Pierre VIGNÉ $^{3}$, Yann DENIAUD ${ }^{1}$}

1. Cerema, Direction Eau, mer et fleuves,

155, rue Pierre Bouguer - BP5, Plouzané, France.

francois.hedou@cerema.fr ; amelie.roche@cerema.fr ; marie.caouissin@cerema.fr ; yann.deniaud@cerema.fr

2. Ministère de la Transition écologique et solidaire, Tour Sequoia, 92055 La Défense CEDEX, France. sabine.moraud@developpement-durable.gouv.fr

3. Cerema, direction Normandie-Centre,

10, chemin de la Poudrière - CS 90245, 76121 Le Grand Quevilly Cedex, France. pierre.vigne@cerema.fr

\section{Résumé :}

Dans le cadre de la Stratégie nationale de gestion intégrée du trait de côte, le Ministère de la Transition écologique et solidaire a demandé au Cerema de produire une cartographie nationale des ouvrages et aménagements susceptibles d'impacter directement l'évolution du trait de côte, tels que les digues, les murs, les jetées et les épis. L'identification et la cartographie de ces ouvrages s'est appuyée sur une campagne nationale de photo-interprétation de séries d'orthophotographies aériennes du littoral. Les ouvrages visibles ont été relevés et leurs principales caractéristiques recensées. Les données collectées ont ensuite été comparées et complétées par des informations issues de bases de données locales accessibles. L'exploitation de ce recensement cartographique met en évidence la présence de près de 16000 ouvrages et aménagements, représentant un linéaire cumulé d'environ 2300 kilomètres, sur les côtes de la France métropolitaine et des départements et régions d'outre-mer. Bien que l'exhaustivité du recensement des ouvrages puisse être limitée par leur visibilité sur les photographies aériennes analysées, cette cartographie apporte néanmoins une première vision homogène du patrimoine des ouvrages littoraux à l'échelle nationale.

\section{Mots-clés :}

Trait de côte, Littoral, Ouvrage, Aménagement, Épi, Digue côtière, Brise-lames, Jetée, Protection, Artificialisation, Dynamiques côtières. 


\section{Thème 6 - Gestion durable des zones littorales et estuariennes}

\section{Introduction}

Les ouvrages et aménagements présents sur le littoral influencent les transits sédimentaires. Leur bonne connaissance est un préalable à la compréhension des dynamiques côtières, paramètre essentiel à la définition de stratégies d'adaptation des territoires aux évolutions du littoral. Si de nombreuses bases de données sur les ouvrages littoraux ont été élaborées pour des besoins locaux de gestion, en s'appuyant parfois sur des préconisations de recensement (ROCHE, 2011), le manque de compatibilité des informations disponibles et leur difficile agrégation pour une exploitation à des échelles plus étendues a conduit à l'inscription de la production d'une cartographie nationale des ouvrages littoraux au programme d'actions 2017-2019 de la Stratégie nationale de gestion intégrée du trait de côte (MEEM, 2017). Cet article a pour objet de présenter la méthode mise en œuvre pour la production de cette couche d'information géographique nationale et les premiers résultats de son exploitation.

\section{Méthodologie}

\subsection{Reconnaissance des ouvrages}

Les formes générales des ouvrages côtiers visibles sur orthophotographies ont été préalablement numérisées au 1/2500 dans le cadre de la cartographie de l'indicateur national de l'érosion côtière (CEREMA et MEDDE, 2015). Ces premières digitalisations ont été complétées et mises à jour avec la photo-interprétation de la BDOrtho HR® de l'IGN dont la grande résolution permet une meilleure reconnaissance des objets recherchés. Les ouvrages et aménagements situés en haut de plage présentant un très faible marnage (façade méditerranéenne et La Réunion) ont également fait l'objet de digitalisations complémentaires. Les informations disponibles dans les bases de données locales des services de l'État sont ensuite analysées afin de compléter les digitalisations. Les zones relevées correspondent à celles étudiées par l'indicateur national de l'érosion côtière et ne concernent donc pas les estuaires, ni l'intérieur des ports abrités par des jetées.

\subsection{Orientation et typologie}

L'orientation (longitudinale ou transversale) et le type d'ouvrages et aménagements littoraux sont renseignés pour chacune des entités digitalisées selon les préconisations de recensement définies par Roche (2011). En complément des orthophotographies disponibles, des images issues de Google Earth ${ }^{\circledR}$ sont exploitées, en particulier dans les secteurs traités avec un modèle numérique de terrain (MNT) précis, afin de faciliter l'interprétation du type des ouvrages rencontrés. Le tableau 1 détaille la typologie des ouvrages et aménagements littoraux digitalisés. 


\section{XVèmes Journées Nationales Génie Côtier - Génie Civil \\ La Rochelle, 29 au 31 mai 2018}

Tableau 1. Typologie des ouvrages et aménagements littoraux digitalisés

\begin{tabular}{|c|c|c|}
\hline Catégorie & Classe & Type \\
\hline \multirow{5}{*}{$\begin{array}{l}\text { Ouvrages de } \\
\text { protection }\end{array}$} & \multirow{3}{*}{$\begin{array}{l}\text { Ouvrage se } \\
\text { substituant au } \\
\text { trait de côte }\end{array}$} & Digue côtière \\
\hline & & Mur, mur de soutènement \\
\hline & & Perré \\
\hline & \multirow{2}{*}{$\begin{array}{l}\text { Ouvrage de } \\
\text { lutte contre } \\
\text { l'érosion }\end{array}$} & Brise-lames \\
\hline & & Épi \\
\hline \multirow{9}{*}{$\begin{array}{l}\text { Autres } \\
\text { aménagements }\end{array}$} & \multirow[t]{2}{*}{ Accès } & Accès, chemin, voie submersible... \\
\hline & & Cale \\
\hline & \multirow[t]{2}{*}{ Bâti } & Bâtiment, blockhaus, fortification... \\
\hline & & Protection individuelle \\
\hline & \multirow{2}{*}{$\begin{array}{l}\text { Infrastructure } \\
\text { portuaire et de } \\
\text { navigation }\end{array}$} & Jetée \\
\hline & & Quai \\
\hline & \multirow[t]{3}{*}{ Divers } & Aménagement hydraulique (vanne, écluse, barrage...) \\
\hline & & $\begin{array}{l}\text { Aménagement de sécurité (poste de secours, } \\
\text { signalisation...) }\end{array}$ \\
\hline & & Autre ou indéterminé \\
\hline
\end{tabular}

\subsection{Date de visibilité}

À défaut de disposer d'informations accessibles sur la date de construction des ouvrages, les années de première et de dernière visibilité sont recherchées sur les orthophotographies disponibles. Les principaux supports utilisés pour cette analyse

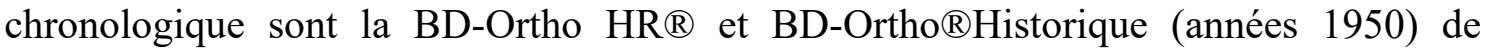
l'IGN, l'Ortholittorale V1 (2000) et V2 (2010-2014) et les archives des années 19201950 traitées par le SHOM et l'Ifremer (C SHOM, Ifremer, photothèque nationale).

\section{Résultats}

3.1. Caractérisation des ouvrages et aménagements littoraux cartographiés

La cartographie des ouvrages et aménagements littoraux concerne la métropole et les 5 départements et régions d'outre-mer. Elle est consultable et téléchargeable sur le portail internet Géolittoral (CEREMA et MTES, 2017). La figure 1 présente un extrait de la représentation cartographique prenant en compte la classe des ouvrages dans sa symbologie.

La cartographie positionne 15949 ouvrages visibles sur les photographies aériennes les plus récentes disponibles au moment de l'étude (2006-2015). 


\section{Thème 6 - Gestion durable des zones littorales et estuariennes}

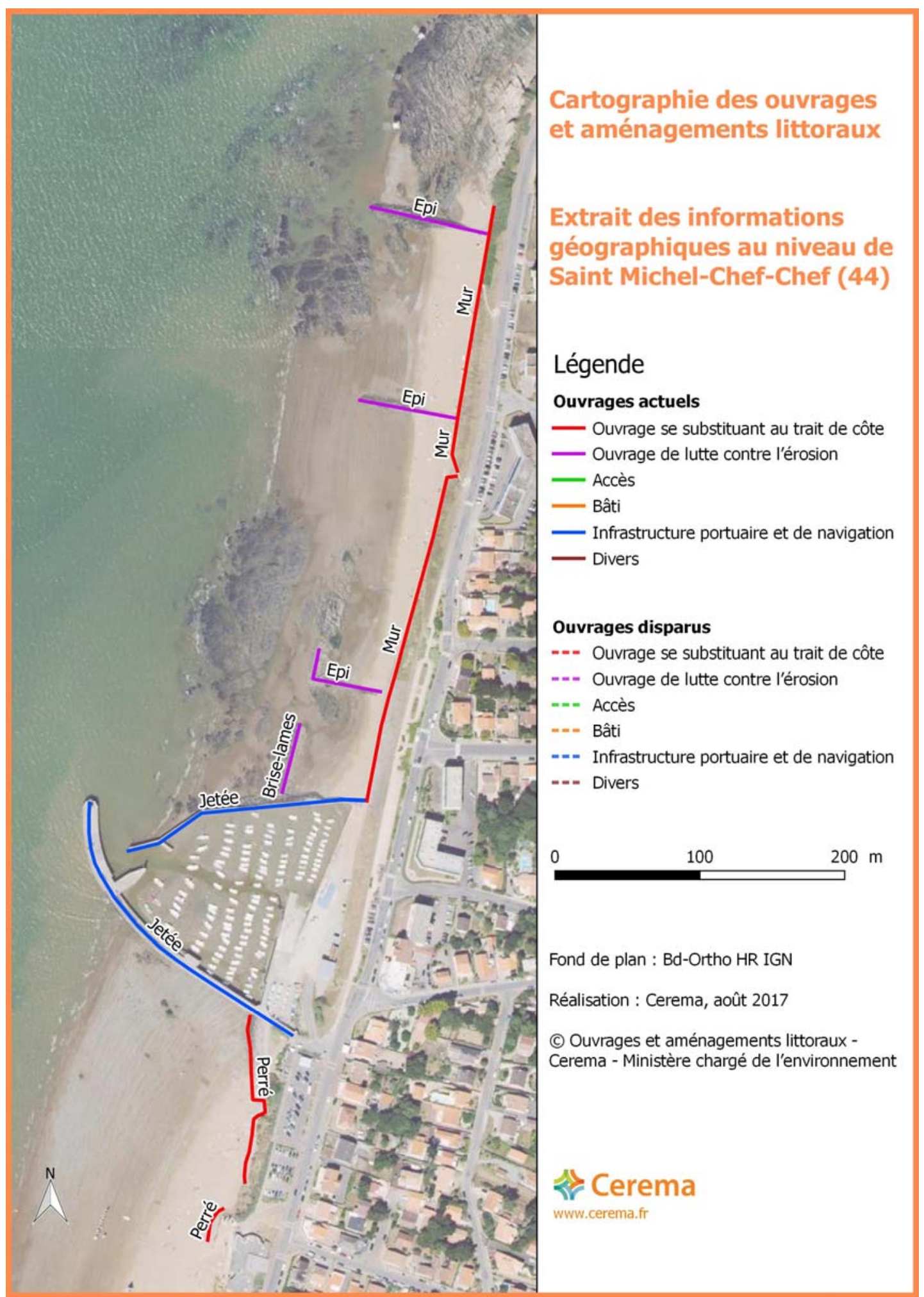

Figure 1. Exemple de relevé des ouvrages et aménagements littoraux à Saint-MichelChef_Chef (fond de plan : BD ORTHO ${ }^{\circledR} H R I G N$ ). 


\section{XVèmes Journées Nationales Génie Côtier - Génie Civil \\ La Rochelle, 29 au 31 mai 2018}

Les ouvrages longitudinaux, avec 10232 entités, sont les plus nombreux et représentent un linéaire cumulé de 1811 kilomètres, soit $79 \%$ du linéaire total digitalisé. Généralement plus courts que les ouvrages longitudinaux, les 5712 ouvrages transversaux recensés cumulent 491 kilomètres (soit $21 \%$ du linéaire total digitalisé).

Les trois types d'ouvrages constituant la classe des ouvrages se substituant au trait de côte (digue, perré et mur, mur de soutènement) représentent à eux seuls un peu plus de la moitié (55\%) des longueurs d'ouvrages recensées dans la cartographie (figure 2). Les épis sont les ouvrages transversaux les plus représentés dans la cartographie (2 091 entités). Avec une longueur cumulée de $115 \mathrm{~km}$, leur longueur moyenne est ainsi estimée à $55 \mathrm{~m}$. Il existe enfin un linéaire significatif d'ouvrages et d'aménagements dont la typologie n'a pas pu être renseignée avec certitude à partir de l'interprétation des photographies aériennes (2 319 entités représentant un linéaire cumulé de $275 \mathrm{~km}$ ).

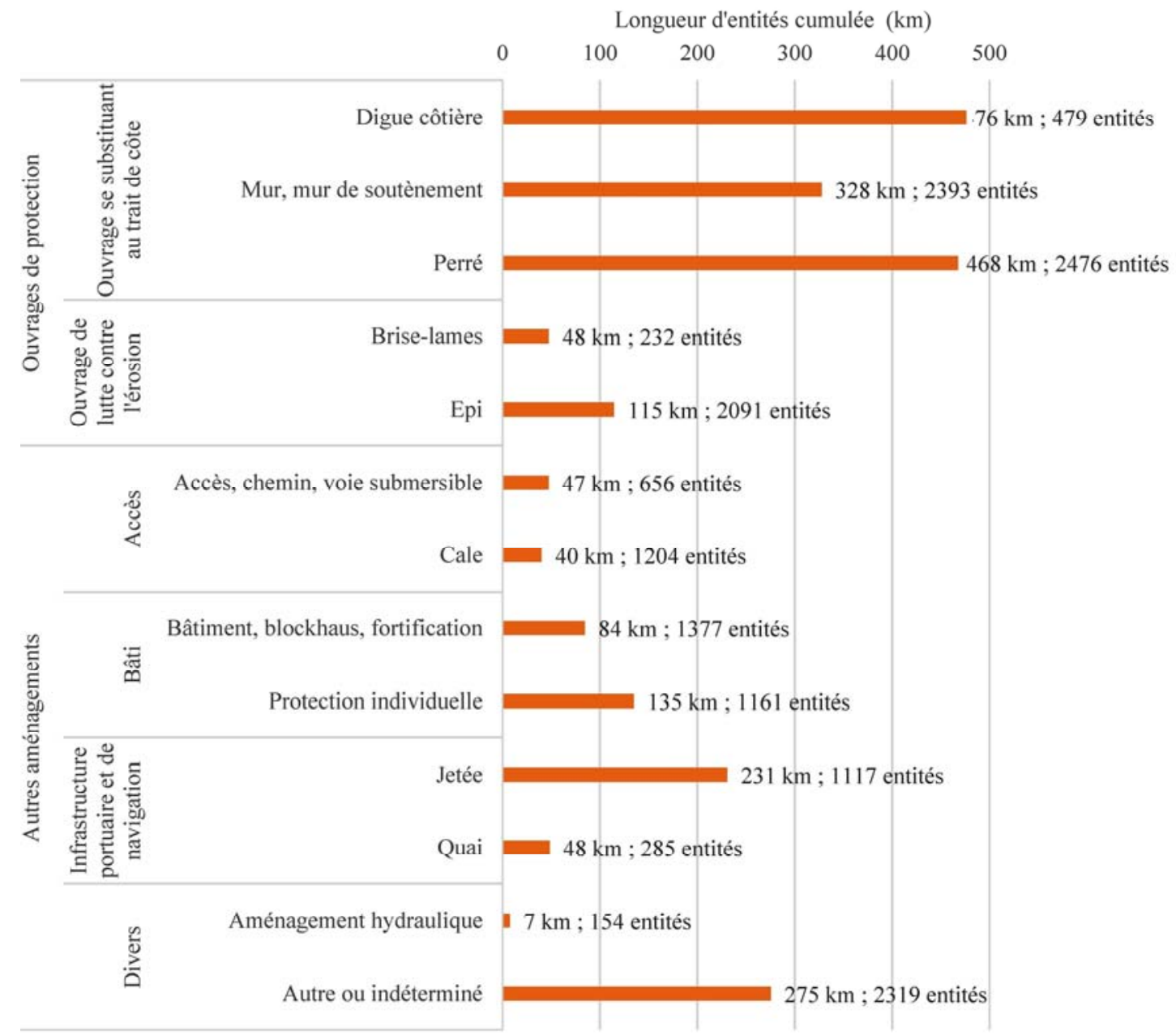

Figure 2. Nombre et longueur cumulée des entités visibles sur les photographies aériennes les plus récentes (2006-2015). 


\section{Thème 6 - Gestion durable des zones littorales et estuariennes}

\subsection{Evolution historique du nombre d'ouvrages et d'aménagements}

L'augmentation de l'artificialisation du trait de côte au cours des dernières décennies est indéniable, mais l'évolution quantitative du nombre et du linéaire d'ouvrages et d'aménagements reste difficile à estimer avec les données utilisées. En raison de la faible résolution des supports anciens (des années 20 aux années 1950), seule l'évolution des ouvrages transversaux, mieux visibles car généralement en contact avec la mer dans tous les contextes hydrographiques côtiers, peut être considérée comme fiable au niveau national. Une augmentation du nombre d'épis (de 379 à 2 091) et de jetées (de 480 à 1 117) a ainsi été constatée entre 1960 et les années 2010.

Au-delà des 15949 ouvrages visibles sur les photographies aériennes les plus récentes, la cartographie comprend 1051 ouvrages et aménagements observés principalement avant les années 1960 mais qui n'apparaissent plus sur les supports récents.

\subsection{Estimation de l'artificialisation du trait de côte}

Le trait de côte peut être considéré comme artificialisé lorsqu'il supporte des ouvrages et des aménagements littoraux ou que son évolution est influencée par ces constructions. Une première estimation du taux artificialisation est réalisée en projetant le tracé des ouvrages et aménagements littoraux sur le trait de côte de référence Histolitt ${ }$. La longueur des ouvrages longitudinaux est directement affectée au tracé de Histolitt ${ }^{\circledR}$. La zone d'influence des ouvrages transversaux est en revanche plus complexe à définir et ne peut être approchée au niveau national qu'à partir d'hypothèses très simplificatrices. Il est ici retenu que le trait de côte est considéré artificialisé sur un linéaire de deux fois la longueur de l'ouvrage transversal réparti de part et d'autre de son point central projeté sur Histolitt®. En compléments sont ajoutés les secteurs portuaires définis lors du projet européen Eurosion (CE, 2004) dont l'emprise n'a pas été recensée dans le cadre des travaux de cartographie des ouvrages et aménagements littoraux.

À partir de ces principes, ce sont de l'ordre de $2900 \mathrm{~km}$ de linéaire de côte qui sont considérés comme impactés par un ouvrage ou par un aménagement côtier ou portuaire. Sur l'emprise étudiée (linéaire affecté d'une nature de trait de côte selon le projet européen Eurosion, soit globalement hors estuaire), le taux d'artificialisation du littoral est ainsi de l'ordre de $35 \%$ en métropole.

\section{Discussion}

\subsection{Exhaustivité de la cartographie}

La qualité des photographies aériennes exploitées (résolution, déformations, contraste), surtout des plus anciennes, n'est parfois pas suffisante pour observer la présence d'un ouvrage sur le littoral. La présence d'ombres portées dans les zones à falaises et de végétation, surtout des arbres de hautes tiges, limite également ponctuellement l'observation des ouvrages. Les méthodes souples (rechargements de plage, systèmes de 


\section{XVèmes Journées Nationales Génie Côtier - Génie Civil \\ La Rochelle, 29 au 31 mai 2018}

drainage de plage, etc.) et les épis recouverts de sable ne peuvent pas, quant à eux, être détectés sur les photographies aériennes. Sans vérification sur le terrain, la cartographie des ouvrages et aménagements littoraux ne peut donc pas être considérée comme exhaustive. Par ailleurs, la faible disponibilité de bases de données locales suffisamment fiables ne permet pas de compenser complètement l'absence de vérification sur site.

\subsection{Qualité des données attributaires}

La résolution des photographies aériennes et des configurations complexes sur le littoral rendent parfois délicates l'interprétation de la typologie des ouvrages et aménagements. Sans autre vérification sur le terrain, celle-ci peut comporter localement des imprécisions. Il en est de même pour les dates de visibilité des ouvrages, en particulier pour les ouvrages longitudinaux souvent difficilement discernables sur les photographies anciennes. Par ailleurs la faiblesse du nombre de séries orthophotographiques disponibles entre les années 1950-60 et 2000 introduit un biais conduisant à retenir des dates de visibilité parfois plus récentes qu'elles ne devraient l'être.

\subsection{Perspectives}

Afin d'améliorer la connaissance des ouvrages et aménagements littoraux, de nouvelles caractéristiques pourraient compléter les données existantes (RANNOU, 2015). La photo-interprétation complétée de quelques contrôles sur site pourrait permettre de renseigner les matériaux de construction ou la largeur des ouvrages. Un croisement avec des MNT précis serait également envisageable pour estimer l'altimétrie de la crête des ouvrages longitudinaux pouvant participer à des systèmes de protection contre les submersions marines. Plus globalement, il apparaît essentiel de poser la question de la mise à jour et de la complétude de la cartographie des ouvrages et aménagements littoraux. Le réseau national des observatoires du trait de côte, qui a vocation à réunir les structures assurant la production et/ou l'utilisation de données relatives au trait de côte, pourrait constituer un lieu d'échanges adapté pour définir ces modalités.

\section{Conclusion}

La cartographie des ouvrages et aménagements littoraux, produite principalement à partir de l'observation de photographies aériennes, apporte des informations sur leur localisation et leurs caractéristiques. Bien que l'usage de la photo-interprétation ne garantisse pas l'exhaustivité du recensement, cette cartographie permet néanmoins d'identifier et de renseigner de manière homogène près de 16000 entités constituant ainsi une première contribution nationale sur les ouvrages et aménagements littoraux. Ces données apportent des connaissances essentielles pour mieux prendre en compte les impacts potentiels des ouvrages et des aménagements dans l'étude des dynamiques sédimentaires à des échelles tant nationale que locale. Leur mise à disposition auprès 


\section{Thème 6 - Gestion durable des zones littorales et estuariennes}

d'un large public a vocation à faciliter l'émergence de choix d'aménagement concertés et adaptés aux évolutions du littoral.

\section{Remerciements}

La réalisation de cette cartographie nationale des ouvrages et aménagements littoraux repose sur l'investissement de 6 directions du Cerema. Les auteurs remercient particulièrement: Raphaël BÉNOT, Nathalie BERENGER, Alexis BERNARD, Bleuenn BODIN, Hervé BOZEC, Florent COLLIN, Noémie COUDRAY, Stéphanie DETOURBE, Agnès GOMEZ, Yan GUIRRIEC, Jean-Philippe HAMON, Magali JOUAS, Neville LE DU, Christelle LESTRÉHAN, Jean-François MAHÉ, Jean-Paul MASSET, Caroline MAURIN, Sandra ORFEO, Frédéric PONS, Kevin RANNOU, Jérôme REVEL, Isabelle ROUX, Vincent VANCRAENENBROECK et Émeric VEDIÉ.

\section{Références bibliographiques}

CE (Commission Européenne) (2004). Living with coastal erosion in Europe: sediment and space for sustainability. Office for Official Publications of the European Communities, Luxembourg. Part I "Major findings and Policy Recommendations of the EUROSION project", 10/05/2004, 54 p.; Part II "Maps and statistics", 29/05/2004, 25 p.

CEREMA (Centre d'études et d'expertise sur les risques, l'environnement, la mobilité et l'aménagement), MEDDE (Ministère de l'Écologie, du Développementdurable et de l'Énergie) (2015). Cartographie de l'indicateur national de l'érosion côtière. Disponible en ligne sur URL: http://www.geolittoral.developpement-durable.gouv.fr/indicateur-national-de-l-erosion-cotierer473.html

CEREMA (Centre d'études et d'expertise sur les risques, l'environnement, la mobilité et l'aménagement), MTES (Ministère de la Transition écologique et solidaire) (2017). Cartographie nationale des ouvrages et aménagements littoraux. Disponible en ligne sur URL : http:/www.geolittoral.developpement-durable.gouv.fr/cartographie-nationale-des-ouvrages-et-r502.html MEEM (Ministère de l'Environnement, de l’Énergie et de la Mer) (2017). Stratégie nationale de gestion intégrée du trait de côte - Programme d'actions 2017-2019. Disponible en ligne sur URL : http://www.geolittoral.developpement-durable.gouv.fr/strategie-nationale-de-gestionintegree-du-trait-r434.html

RANNOU K. (2015). Amélioration de la connaissance des ouvrages côtiers français. Mémoire de stage de Master 2 Sciences de la mer et du Littoral, mention Expertise et gestion de l'environnement littoral. Université de Bretagne occidentale, Brest, 67 p.

ROCHE A. (2011). Préconisations pour le recensement des ouvrages et structures de défense contre les aléas côtiers. Collection Connaissances du Cerema (ex. Cetmef), 62 p. Disponible en ligne sur URL : https://www.cerema.fr/fr/centre-ressources/boutique/preconisationsrecensement-ouvrages-structures-defense 